\title{
Traducción y Validación del Collective Efficacy Questionnaire for Sports (CEQS) en Deportistas Mexicanos
}

\author{
Translation and Validation of the Collective Efficacy Questionnaire for Sports \\ (CEQS) in Mexican Athletes
}

\author{
Julio Román Martínez-Alvarado ${ }^{1}$, Félix Guillén García ${ }^{2}$, Luis Horacio Aguiar Palacios ${ }^{3}$ y Ana \\ Gabriela Magallanes Rodríguez ${ }^{4}$
}

\begin{abstract}
Resumen
El objetivo del presente estudio fue analizar las propiedades psicométricas del Collective Efficacy Questionnaire for Sports (CEQS) con deportistas mexicanos. La muestra estuvo compuesta por 935 deportistas con edades comprendidas entre los 10 y 35 años. Los resultados del Análisis Factorial Confirmatorio (AFC) mostraron un mejor ajuste del modelo de cinco factores del CEQS (CFI=.94; TLI=.91; $\mathrm{IFI}=.94$; RMSEA=.07), comparado con los índices de ajuste del modelo unifactorial (CFI=.91; TLI=.88; IFI=.91; RMSEA=.08). En cuanto a los resultados del análisis de fiabilidad, el CEQS mostró puntuaciones aceptables, tanto por consistencia interna como estabilidad temporal. Los resultados de la comparación de medias por género, asumió varianzas iguales en los cinco factores del CEQS. Se concluye que, en términos generales, el CEQS muestra adecuadas propiedades psicométricas, indicándose su uso para evaluar la eficacia colectiva en el contexto del deporte con muestra mexicana.
\end{abstract}

Palabras clave: eficacia colectiva, CEQS, análisis factorial confirmatorio, estabilidad temporal

\begin{abstract}
The objective of the present study was to analyze the psychometric properties of the CEQS with Mexican athletes. The sample consisted of 935 athletes aged between 10 and 35 years. The results of the Confirmatory Factor Analysis (CFA) showed a better fit for a five-factor model of the CEQS (CFI=.94, TLI=.91, IFI=.94, RMSEA=.07), compared with the adjustment indices of the unifactorial model (CFI=. 91; TLI=.88; IFI=.91; RMSEA=.08). The results of the reliability analysis showed acceptable scores for both internal consistency and temporal stability. The comparison of means by gender showed equal variances in the five factors. In conclusion, the CEQS shows adequate psychometric properties indicating its use to evaluate the collective efficacy in the context of sport with a Mexican sample.
\end{abstract}

Keywords: collective efficacy, CEQS, confirmatory factor analysis, temporal stability

\footnotetext{
${ }^{1}$ Doctor en Psicología del Deporte y la Actividad Física. Profesor Investigador. Universidad Autónoma de Baja California. Calle tecate 20174, Colonia Buenos Aires. C.P 22536, Argentina. Tel.: (664)1260977. Correo: jmartinez2@uabc.edu.mx

${ }^{2}$ Doctor en Psicología. Profesor Titular Universidad. Universidad de Las Palmas de Gran Canaria. Santa Juana de Arco, 1. 35004 Las Palmas de Gran Canaria, España. Tel.: 627932090. Correo: felix.guillen@ulpgc.es

${ }^{3}$ Maestría en Ciencias de la Salud. PTC, Responsable de Servicio Social Profesional. Escuela de Ciencias de la Salud, Valle de las Palmas, UABC. Blvd Universitario 1000, Valle de Las Palmas, 21500, Tijuana, B.C., México. Tel.: 6461178344.

Correo: aguiar.luis@uabc.edu.mx

${ }^{4}$ Doctora en Ciencias de la Salud. Escuela de Ciencias de la Salud, Valle de las Palmas, Universidad Autónoma de Baja California, Bugambilias \#50, Interior 301, fraccionamiento El Prado,Tijuana, Baja California, México. CP 22440. Tel.: (664) 3053123. Correo: gabriela,magallanes@uabc.edu.mx
}

Revista Iberoamericana de Diagnóstico y Evaluación - e Avaliação Psicológica. RIDEP · №54 · Vol.1 · 47-55 • 2020

ISSN: 1135-3848 print /2183-6051online 


\section{Introducción}

Los deportes de equipo requieren una alta colaboración entre sus miembros, siendo necesario trabajar juntos, de manera coordinada en la ejecución de tareas en beneficio del equipo $\mathrm{y}$, en algunas ocasiones, dichas tareas se deben ejecutar bajo presión y con pocas interrupciones. Esta interdependencia donde los miembros del equipo trabajan como una unidad (Chow \& Feltz, 2008), no es fácil de conseguir ya que intervienen diferentes factores, de los cuales queremos destacar la percepción de eficacia colectiva, concepto abordado por Bandura (1997). Los integrantes de un equipo comparten un sistema de creencias, mismo que contribuye en la organización durante la ejecución de tareas. A este sistema de creencias en común se le denomina eficacia colectiva, concepto emergido dentro del marco conceptual de la teoría social cognitiva. Bandura (1997) afirma que la eficacia colectiva es la "creencia compartida de un grupo en su capacidad conjunta para organizar y ejecutar las acciones necesarias para alcanzar determinados niveles de logro" (p. 476).

A diferencia de la eficacia individual, la eficacia colectiva implica una dinámica social interactiva, coordinada y sinérgica (Fernández, Díez, Caprara, Barbaranelli, \& Bandura, 2002). Los equipos que se perciben como más eficaces, tendrán aspiraciones colectivas más altas en comparación con los equipos con baja eficacia colectiva; por lo que es probable que su capacidad de resistir ante los obstáculos, sea mayor (Bandura, 2001). Los equipos con alta eficacia colectiva serán persistentes al mantener cursos de acción ante una adversidad, por lo tanto, preferirán una estrategia de mayor compromiso y la intensificación del esfuerzo, mientras que los equipos de baja eficacia colectiva, buscarán apaciguar el compromiso al hacerle frente a las adversidades (Lee \& Farh, 2004).

Existen diversos estudios que se han encargado de analizar la asociación entre la eficacia colectiva y constructos como la cohesión grupal (Heuzé, Raimbault, \& Fontayne, 2006; Leo, Sánchez-Miguel, Sanchez-Oliva, Amado, \& García-Calvo, 2011; Martínez \& Ciruelos, 2013), los conflictos de equipo (Leo, González-Ponce, Sánchez-Miguel, Ivarsson, \& García-Calvo,
2015), la comunicación intercultural (Bell \& Riol, 2017), los modelos mentales de equipo (Filho, Tenenbaum, \& Yang, 2015), las emociones (Petitta \& Jiang, 2015), la autoeficacia (Magyar, Feltz, \& Simpson, 2004) el comportamiento del entrenador durante la competencia (Atkinson, Short, \& Martin, 2018; Høigaard, Cuyper, Fransen, Boen, \& Peters, 2015) y el rendimiento deportivo (Blecharz et al., 2014; Heuzé, Sarrazin, Masiero, Raimbault, \& Thomas, 2006; Myers, Feltz, \& Short, 2004). A pesar de la gran variedad de constructos asociados a la eficacia colectiva en el contexto deportivo, es difícil comparar resultados ya que se han utilizado diferentes instrumentos para evaluar la eficacia colectiva, siendo la metodología predominante la construcción de instrumentos específicos por tipo de deporte (Feltz, Short, \& Sullivan, 2008). En este sentido, se puede encontrar en la literatura un número considerable de investigaciones que utilizan estas mediciones específicas (Chow \& Feltz, 2008; Feltz \& Lirgg, 1998; Heuzé, Raimbault et al., 2006; Heuzé, Sarrazin et al., 2006; Magyar et al., 2004; Myers et al., 2004). Siguiendo esta línea, Bandura (2006) hace una serie de recomendaciones para la construcción de escalas de eficacia, destacando la importancia de adecuar al dominio, lo cual es posible incluyendo items que reflejen tareas concretas del deporte a evaluar. Finalmente, los ítems se seleccionan consultando con expertos del deporte para el cual se está desarrollando la escala.

Por otra parte, existe un segundo enfoque que propone la utilización de medidas globales o generales para evaluar la eficacia colectiva. En este sentido Short, Sullivan y Feltz (2005) desarrollaron una medida global de eficacia colectiva en el deporte, la cual fue denominada Collective Efficacy Questionnaire for Sports (CEQS). El CEQS fue elaborado pensando en que fuera una medida multidimensional de la eficacia colectiva consistente con la afirmación de Bandura (1997) de que "las creencias de eficacia involucran diferentes tipos de capacidades, como el manejo del pensamiento, el afecto, la acción y la motivación" (p. 45). El CEQS es una medida multidimensional de la eficacia colectiva que se compone por 20 ítems, distribuidos equitativamente en cinco factores: habilidad, preparación, esfuerzo, persistencia y unión. Estos 
factores hacen referencia a diferentes aspectos del funcionamiento general de los equipos y que son importantes para la eficacia colectiva (Short et al., 2005). La habilidad representa la capacidad que los deportistas creen tener para realizar las acciones necesarias durante un partido $o$ competencia. El factor preparación manifiesta el convencimiento del deportista de que su equipo reúne una adecuada preparación de los aspectos fundamentales para el buen desempeño durante la competencia (preparación física, psicológica y estratégica). El esfuerzo refleja el conjunto de creencias relacionadas con la capacidad colectiva para emplear gran cantidad de trabajo en superar situaciones adversas propias de la competencia. El factor persistencia hace referencia a la capacidad del equipo para mantenerse firme durante situaciones críticas específicas de la competencia. La unión representa la creencia en que el equipo sea capaz de resolver conflictos, mantener actitudes positivas y establecer una comunicación efectiva. Los autores del CEQS contemplaron una medida global de la eficacia colectiva que puede calcularse sumando las puntuaciones de los 20 ítems del cuestionario.

El CEQS ha demostrado adecuadas propiedades psicométricas con deportistas estadounidenses (Short et al., 2005), españoles (Martínez, Guillén, \& Feltz, 2011), brasileños (Paes, 2014), japoneses (Uchida, Machida, Tsuchiya, \& Kugihara, 2014) o turcos (Öncü, Feltz, Lirgg, \& Gürbüz, 2018). En su estudio original short et al., (2005) obtuvieron respaldo a la validez de constructo, convergente, predictiva y discriminante del CEQS, así como de su consistencia interna. Diferentes investigaciones han realizado estudios de adaptación y validación del CEQS (Martínez et al., 2011; Öncü et al., 2018; Paes, 2014; Uchida et al., 2014), para lo cual se han utilizando deportistas de diferentes deportes y niveles de rendimiento, encontrando respaldo a la fiabilidad y validez del cuestionario. Estos estudios confirmaron la estructura de cinco factores, con la misma distribución de los ítems. En la adaptación japonesa del CEQS (denominada J-CEQS) se utilizó una muestra de 1244 deportistas. Resultados similares se encontraron con 312 deportistas españoles (Martínez et al., 2011).

La utilización de escalas globales para la medición de la eficacia colectiva permitiría mayor flexibilidad y funcionalidad al facilitar la recopilación de aspectos generalizables a varios deportes, en lugar de concentrarse en aspectos particulares de un deporte, como ocurre con las escalas específicas. El uso de una medida global, como el CEQS, puede proporcionar mayores ventajas a la hora de explorar la compleja red de variables asociadas con la eficacia colectiva, ya que un solo estudio podría aplicarse en más de un deporte, permitiendo elaborar análisis comparativos en función del mismo.

Por otro lado, el CEQS podría convertirse en una herramienta facilitadora de los proceso de evaluación, sensible a elementos del contexto sociocultural (Casullo, 1999) del deportista, haciendo más eficaz la evaluación psicológica. Esto cobra relevancia al tratarse de un elemento que, al igual que la autoconfianza, está íntimamente relacionado con la ejecución y las estrategias que utiliza el deportista para resolver situaciones de competición (Fradejas, Espada, \& Garrido, 2017). Con base en lo anterior, el propósito de la presente investigación es analizar las propiedades psicométricas del Collective Efficacy Questionnaire for Sports (CEQS) con deportistas mexicanos.

\section{Método}

\section{Participantes}

La muestra estuvo compuesta por 935 deportistas federados de la ciudad de Tijuana, de ambos sexos (Hombres $=69 \%$, mujeres $=31 \%$ ) y con edades comprendidas entre los 10 y 35 años $(M=18.9, D T=3.79)$. Los participantes reportaron practicar algún deporte de equipo (Fútbol $=54 \%$, fútbol americano $=24 \%$, fútbol bandera $=20 \% \mathrm{y}$ baloncesto $=2 \%$ ), llevar en promedio 5.3 años jugando su deporte y un promedio de 1 año afiliado a su equipo. Finalmente, estos deportistas reportan entrenar una media de 6.62 horas a la semana.

\section{Procedimiento}

Para la traducción del instrumento se utilizó la metodología parallel back-translation (Brislin, 1986), en la que dos traductores profesionales tradujeron la versión original en inglés del CEQS 
al español y otros dos traductores realizaron la traducción español-inglés sin conocer la versión original del cuestionario. Posteriormente los investigadores analizaron las cuatro versiones del cuestionario, siendo la principal tarea mantener el sentido original de cada ítem. El resultado final de estas traducciones fue la versión preliminar del CEQS adaptado al español mexicano, la cual consideró aspectos semánticos de esta cultura con el fin de conseguir mejor entendimiento de cada ítem.

El cuestionario fue aplicado de forma colectiva y auto administrada una hora antes del partido ya que por la naturaleza del constructo a medir, se recomienda hacer la medición lo más cercano al momento de la competición. El número reducido de ítems del CEQS permitió que la duración para contestar no excediera los 10 minutos, por lo que el funcionamiento pre-partido de los equipos no fue alterado. Se enfatizó la confidencialidad de los datos y en la participación voluntaria de los deportistas. Todos los participantes entregaron al investigador principal consentimiento informado de participación.

\section{Instrumento}

Eficacia colectiva. Para la medición de la eficacia colectiva utilizamos el Collective Efficacy Questionnaire for Sports (Short et al., 2005). El CEQS presenta una estructura factorial conformada por cinco elementos que evalúan diferentes aspectos de la eficacia colectiva en el deporte: esfuerzo, capacidad, preparación, persistencia y unidad. Cada factor se compone por cuatro ítems redactados de forma clara, breve y que expresan un juicio de capacidad en el presente y no sus posibilidades futuras. El CESQ utiliza una escala tipo Likert de once puntos (del 0 al 10) que puntúa las respuestas desde "No confía en absoluto" hasta "Confía de manera extrema", empleando ítems que representan diferentes niveles de exigencia en las tareas colectivas. Las instrucciones iniciales reflejan la confianza del equipo en su capacidad frente a una situación de competencia próxima a enfrentar ("Puntúa en qué medida tu equipo confía sobre lo que es capaz, ante un partido o competición inminente..."). Un ejemplo de ítem por factor sería: "Jugar al máximo de sus capacidades" (Esfuerzo), "Persistir cuando se presentan obstáculos" (Persistencia), "Estar listo" (Preparación), "Estar unidos"
(Unidad) y "Jugar con más habilidad que el oponente" (Habilidad).

\section{Análisis de datos}

Utilizando el programa estadístico SPSS 23.0, se realizó un análisis de los estadísticos descriptivos (Media, desviación típica, asimetría y curtosis) de los 20 ítems del CEQS, con el fin de estudiar el grado en que los datos se asemejaban a la normalidad estadística. Para evaluar la validez de constructo se efectuó un análisis de correlación de Pearson entre las cinco subescalas del cuestionario. Se estudió la fiabilidad del CEQS en cuanto a consistencia interna, utilizando el coeficiente alfa de Cronbach y la estabilidad temporal a través de la técnica test-retest.

Para el estudio de la estructura factorial del CEQS, se utilizó el Análisis Factorial Confirmatorio (AFC), a través del programa AMOS 23.0. Se analizó el ajuste de diferentes modelos donde se incluía el modelo original del CEQS de cinco factores y un modelo unifactorial alterno. Para comprobar la bondad de ajuste de los modelos hipotetizados, se emplearon diferentes índices: El Índice Comparativo de Ajuste (CFI), el Índice de Tucker-Lewis (TLI), el Índice de Ajuste Incremental (IFI) y el Error de la Raíz Cuadrada Media de Aproximación (RMSEA). Finalmente se utilizó la Prueba $t$ de student para la comparación de medias por género de las cinco subescalas del CEQS.

\section{Resultados}

\section{Análisis descriptivo de los ítems del CEQS}

En la Tabla 1 se muestran los estadísticos descriptivos de los 20 ítems del CEQS. Como se puede apreciar, las medias de los ítems oscilan entre 7.76 y 8.30 , lo cual indica que los deportistas presentan niveles relativamente altos en eficacia colectiva. En cuanto a la desviación típica en general encontramos resultados dentro de los parámetros $(D T<2)$. Las puntuaciones en asimetría y curtosis se encontraron dentro de la normalidad estadística. De acuerdo con los criterios de normalidad univariada (Curran, West, \& Finch, 1996), para que se cumpla con dicha normalidad, asimetría debe mostrarse por debajo del valor absoluto 2 y curtosis por debajo del valor absoluto 7. En base a estos resultados, se recomienda continuar con el análisis factorial de los ítems. 
Tabla 1. Estadísticos descriptivos para los ítems del CEQS

\begin{tabular}{lcccc}
\hline Ítem & $M$ & $D T$ & Asimetría & Curtosis \\
\hline Superar en jugadas al equipo rival. & 8.05 & 1.80 & -.98 & .54 \\
Resolver conflictos. & 7.67 & 1.69 & -.74 & .49 \\
Actuar bajo presión. & 7.55 & 1.87 & -.82 & .48 \\
Estar listo. & 8.00 & 1.74 & -.91 & .57 \\
Mostrar más habilidad que el otro equipo. & 8.00 & 1.78 & -1.02 & 1.21 \\
Estar unidos. & 8.01 & 1.89 & -1.19 & 1.63 \\
Persistir cuando se presentan obstáculos. & 7.91 & 1.77 & -1.06 & 1.25 \\
Demostrar una fuerte ética de equipo. & 8.01 & 1.80 & -1.00 & .80 \\
Permanecer en el juego cuando parece que tu equipo no tiene descanso alguno. & 7.91 & 1.95 & -1.10 & .81 \\
Jugar al máximo de su capacidad. & 8.14 & 1.83 & -1.34 & 2.14 \\
Jugar bien sin su jugador "estrella". & 8.07 & 1.82 & -1.09 & .96 \\
Prepararse mentalmente para la competencia. & 8.02 & 1.84 & -1.25 & 1.91 \\
Mantener una actitud positiva. & 8.14 & 1.79 & -1.32 & 2.06 \\
Jugar con más habilidad que el oponente. & 8.02 & 1.85 & -1.42 & 2.67 \\
Jugar mejor que el equipo contrario. & 8.04 & 1.85 & -1.30 & 1.88 \\
Mostrar entusiasmo. & 8.16 & 1.81 & -1.27 & 1.86 \\
Superar distracciones. & 7.71 & 2.05 & -1.08 & .82 \\
Prepararse físicamente para esta competencia. & 7.97 & 2.06 & -1.33 & 1.73 \\
Preparar una estrategia exitosa. & 7.96 & 1.92 & -1.27 & 1.51 \\
Mantener una comunicación efectiva. & 8.16 & 1.92 & -1.23 & 1.19 \\
\hline
\end{tabular}

Tabla 2. Análisis de fiabilidad y de correlación de las subescalas del CEQS

\begin{tabular}{|c|c|c|c|c|c|c|c|c|c|}
\hline & & $\alpha$ & $\begin{array}{l}\text { Test- } \\
\text { retest }\end{array}$ & 1 & 2 & 3 & 4 & 5 & 6 \\
\hline 1 & Esfuerzo & .81 & .96 & & & & & & \\
\hline 2 & Persistente & .83 & .95 & $.80 * *$ & & & & & \\
\hline 3 & Preparación & .81 & .97 & $.83^{* *}$ & $.78 * *$ & & & & \\
\hline 4 & Unidad & .79 & .71 & $.83 * *$ & $.81 * *$ & $.81 * *$ & & & \\
\hline 5 & Habilidad & .83 & .96 & $.77 * *$ & $.76^{* *}$ & $.76^{* *}$ & $.78 * *$ & & \\
\hline 6 & CEQS total & .95 & .96 & $.93 * *$ & $.91 * *$ & $.92 * *$ & $.92 * *$ & $.89 * *$ & \\
\hline
\end{tabular}

\section{Análisis de correlación y de fiabilidad de las subescalas del CEQS}

Como se puede observar en la Tabla 2, las puntuaciones en el coeficiente de correlación son significativas y positivas en todos los casos, obteniendo valores entre .71 y $.92(p<.01)$, siendo la relación más significativa la que se dio entre el factor esfuerzo y CEQS total $(r=.93, p<.01)$.

En cuanto el análisis de la fiabilidad por el método de consistencia interna, los resultados mostraron puntuaciones en alfa de Cronbach superiores a .70 en todos los casos, siendo la puntuación más elevada la obtenida por las subescalas de habilidad y persistencia $(\alpha=.83)$. De acuerdo con Nunnally (1978), valores alfa iguales o superiores a .70, se consideran aceptables.

Para evaluar la estabilidad temporal del CEQS, se utilizó una submuestra de 111 deportistas mexicanos, de ambos sexos (Hombres $=87 \%$, mujeres $=13 \%)$ y con edades comprendidas entre los 10 y 33 años $(M=20.2$,
$D T=4.89)$. Estos deportistas reportaron 4.55 años $(D T=5.00)$ de práctica deportiva y una media de $6.91(D T=4.11)$ horas de entrenamiento semanal. El tiempo considerado entre las dos mediciones fue 10 días como mínimo. Los resultados indicaron alta estabilidad temporal en todos los casos, encontrando correlaciones entre .90 y .96 .

\section{Análisis Factorial Confirmatorio}

Se compararon dos modelos hipotéticos de la estructura factorial del CEQS a través de un AFC de máxima verosimilitud. En el modelo 1 se probó la estructura factorial original del CEQS de cinco factores. Por otro lado, en el modelo 2 se evaluó una estructura unifactorial, tomando en consideración las altas correlaciones entre los factores del CEQS. Los resultados del AFC muestran un mejor ajuste del modelo 1 , obteniendo índices de ajuste satisfactorios en todos los casos (TLI=.91， IFI=.94, CFI=.94 y RMSEA=.07), encontrándose los valores de RMSEA dentro del 
Tabla 3. Cargas factoriales estandarizadas

\begin{tabular}{lccccc}
\hline Ítem & Esfuerzo & Persistencia & Preparación & Unidad & Habilidad \\
\hline 8 & .78 & & & & \\
10 & .76 & & & & \\
16 & .74 & & & & \\
17 & .63 & .64 & & & \\
3 & & .79 & & & \\
7 & & .78 & & & \\
9 & & .75 & .73 & & \\
11 & & & .71 & & \\
4 & & & .67 & & \\
12 & & & & .71 & \\
18 & & & & .74 & \\
19 & & & & .75 & \\
2 & & & & & \\
6 & & & & & \\
13 & & & & & \\
20 & & & & & \\
1 & & & & & \\
5 & & & & & \\
14 & & & & & \\
15 & & & & & \\
\hline
\end{tabular}

Tabla 4. Prueba t de student de muestras independientes

\begin{tabular}{lcccc}
\hline & \multirow{2}{*}{$t$} & \multirow{2}{*}{ Valor $p$} & \multicolumn{2}{c}{ 95\% Intervalo de confianza para la diferencia } \\
\cline { 4 - 5 } & .48 & .634 & Inferior & Superior \\
\hline Esfuerzo & .71 & .478 & -.16 & .26 \\
Persistencia & .31 & .754 & -.13 & .29 \\
Preparación & .03 & .973 & -.18 & .24 \\
Unidad & 2.21 & .027 & -.20 & .20 \\
Habilidad & .83 & .407 & .03 & .44 \\
CEQS Total & & & -.11 & .27 \\
\hline
\end{tabular}

intervalo que va de .060 a .079 . En el modelo 2 (Unifactorial) se encontraron índices de ajuste apenas aceptables (TLI=.88, IFI=.91, CFI=.91 y RMSEA=.08), por lo que se decidió apoyar la estructura de cinco factores.

En la Tabla 3 se observan las estimaciones de cada uno de los ítems que componen el CEQS. Los resultados muestran cargas factoriales aceptables en todos los ítems, oscilando estos valores entre .62 у .79 .

\section{Diferencias de género}

La Tabla 4 muestra los resultados de la prueba $\mathrm{t}$ de student para diferenciación por género en cuanto a las puntuaciones de los cinco factores del CEQS. De acuerdo a estos resultados, se asumen varianzas iguales por género no existiendo ninguna diferencia siginificativa.

\section{Discusión}

El objetivo de la presente investigación ha sido analizar las propiedades psicométricas del
Collective Efficacy Questionnaire for Sports (CEQS) con deportistas mexicanos. De acuerdo a los resultados obtenidos de los diferentes análisis, la estructura pentafactorial del CEQS es adecuada. Estos resultados coinciden con otros estudios donde el CEQS muestra adecuadas propiedades psicométricas (Martínez et al., 2011; Paes, 2014; Short et al., 2005; Uchida et al., 2014). Respecto al estudio de la fiabilidad del CEQS, encontramos resultados satisfactorios en el análisis de la consistencia interna y la estabilidad temporal, por lo que se puede afirmar que el cuestionario es fiable.

Para el estudio de la validez de constructo, primeramente, se elaboró un análisis de correlación bivariada entre los factores del CEQS, hipotetizando que las relaciones serían significativa y positiva. En segundo lugar, se procedió al Análisis Factorial Confirmatorio. De acuerdo a los resultados obtenidos del análisis de correlación, las subescalas del CEQS se relacionaron positiva y significativamente. En cuanto a los resultados del AFC, se obtuvo 
respaldo a la estructura de cinco factores del CEQS, presentando mejor ajuste que el modelo unifactorial. Se destaca así la importancia de mantener los cinco elementos de la eficacia colectiva de manera diferenciada, que permiten evaluar diferentes facetas del constructo. Continuando con el análisis de los resultados del AFC, se encontraron cargas factoriales aceptables, lo cual indica que todos los ítems contribuyen de manera significativa a la medición de la eficacia colectiva. Esto adquiere especial relevancia al considerar que se trata de una traducción del inglés al español donde se hicieron varios ajustes a los ítems, con el fin de que resultara comprensible para los deportistas mexicanos lo que cada ítem intentaba expresar. En su mayoría, el CEQS contempla ítems que expresan tareas propias de los deportes de equipo, incluyendo diferentes grados de dificultad en la realización de tareas, por lo que se vuelve complejo adaptar a otros idiomas.

En futuras investigaciones sería conveniente estudiar la validez predictiva, utilizando cuestionarios de constructos de equipo como puede ser la comunicación eficaz intragrupo, la cohesión grupal, la cooperación de equipo o los modelos mentales de equipo. Además, se considera necesario la utilización de muestra heterogénea en cuanto al tipo de deporte y el nivel de rendimiento.

Finalmente, como conclusión principal, los resultados de la presente investigación respaldan la utilización del CEQS para la evaluación de la eficacia colectiva en el contexto deportivo con muestra mexicana; lo que por consecuencia aumenta la probabilidad de que investigadores interesados en el estudio de la eficacia colectiva en el deporte puedan desarrollar mayor investigación en un corto plazo.

\section{Referencias}

Atkinson, F., Short, S. E., \& Martin, J. (2018). College soccer players' perceptions of coach and team efficacy. The Sport Psychologist, 32(3), 237-243. doi.org/10.1123/tsp.2017-0066

Bandura, A. (1997). Self-efficacy: The exercise of control. San Francisco, CA: Freeman.
Bandura, A. (2001). Social cognitive theory of mass communication. Media Psychology, 3, 265-299.

doi.org/10.1207/S1532785XMEP0303_03

Bandura, A. (2006). Guide for creating selfefficacy scales. En F. Pajares y T. Urdan (Eds.), Self-efficacy beliefs of adolescents (pp. 307-337). Greenwich, CT: Information Age.

Bell, R., \& Riol, C. F. (2017). The impact of cross-cultural communication on collective efficacy in NCAA basketball teams. International Journal of Cross Cultural Management, $\quad$ 17(2), 175-195. doi:10.1177/1470595817702678

Blecharz, J., Luszczynska, A., Scholz, U., Schwarzer, R., Siekanska, M., \& Cieslak, R. (2014). Predicting performance and performance satisfaction: Mindfulness and beliefs about the ability to deal with social barriers in sport. Anxiety, Stress, \& Coping, 27(3), 270-287. doi:10.1080/10615806.2013.839989

Brislin, R. W. (1986). The wording and translation of research instruments. In W. J. Lonner \& J. W. Berry (Eds.), Field methods in cross-cultural research (pp. 137-164). Beverly Hills, CA: Sage.

Casullo, M. M. (1999). La evaluación psicológica: Modelos, técnicas y contexto sociocultural. Revista Iberoamericana de Diagnóstico y Evaluación - e Avaliação Psicológica, 7(1), 97-113.

Chow, G. M., \& Feltz, D. L. (2008). Exploring a relationship between collective efficacy, perceptions of success, and team attributions. Journal of Sport Sciences, 26(11), 1179-1189. doi:10.1080/02640410802101827

Curran, P. J., West, S. G., \& Finch, J. F. (1996). The robustness of test statistics to nonnormality and specification error in confirmatory factor analysis. Psychological Methods, 1(1), 16-29.

doi:10.1037/1082-989x.1.1.16

Feltz, D. L., \& Lirgg, C. D. (1998). Perceived team and player efficacy in hockey. Journal of Applied Sport Psychology, 83, 557-564. doi:10.1037/0021-9010.83.4.557

Feltz, D. L., Short, S. E., \& Sullivan, P. J. (2008). Self-efficacy in sport: Research and Strategies 
for working with athletes, teams, and coaches. Champaign, IL: Human Kinetic.

Fernández, R., Díez, J., Caprara, G. V., Barbaranelli, C., \& Bandura, A. (2002). Determinants and structural relation of personal efficacy to collective efficacy. Applied psychology: An international review, 51(1), 107-125.

doi.org/10.1111/1464-0597.00081

Filho, E., Tenenbaum, G., \& Yang, Y. (2015). Cohesion, team mental models, and collective efficacy: Towards an integrated framework of team dynamics in sport. Journal of Sports Sciences, $\quad 33(6), \quad 641-653$. doi:10.1080/02640414.2014.957714

Fradejas, E., Espada, M., \& Garrido, R. (2017). La autoconfianza en el deporte en edad escolar. Revista Iberoamericana de Diagnóstico y Evaluación - e Avaliação Psicológica, $\quad 44(2), \quad$ 158-171. doi.org/10.21865/RIDEP44.2.13

Heuzé, J. P., Raimbault, N., \& Fontayne, P. (2006). Relationships between cohesion, collective efficacy and performance in professional basketball teams: An examination of mediating effects. Journal of Sports Sciences, 24, 59-68. doi: $10.1080 / 02640410500127736$

Heuzé, J. P., Sarrazin, P., Masiero, M., Raimbault, N., \& Thomas, J. P. (2006). The relationships of perceived motivational climate to cohesion and collective efficacy in elite female teams. Journal of Applied Sport Psychology, 18, 201218. doi.org/10.1080/10413200600830273

Høigaard, R., De Cuyper, B., Fransen, K., Boen, F., \& Peters, D. M. (2015). Perceived coach behavior in training and competition predicts collective efficacy in female elite handball players. International Journal of Sport Psychology, 46(6), 321-336. doi:10.7352/IJSP2015.46.321

Lee, C., \& Farh, J. L. (2004). Joint effects of group efficacy and gender diversity on group cohesion and performance. Applied Psychology: An International Review, 53(1), 136-154. doi.org/10.1111/j.1464-0597.2004.00164.x

Leo, F. M., González-Ponce, I., Sánchez-Miguel, P. A., Ivarsson, A., \& García-Calvo, T. (2015). Role ambiguity, role conflicto, team conflicto, cohesion and collective efficacy in sport teams: A multilevel analysis. Psychology of Sport and Exercise, 20, 60-66. doi.org/10.1016/j.psychsport.2015.04.009

Leo, F. M., Sánchez-Miguel, P. A., SánchezOliva, D., Amado, D., \& García-Calvo, T. (2011). Incidencia de la cooperación, la cohesión y la eficacia colectiva en el rendimiento en equipos de fútbol. Revista Internacional de Ciencias del Deporte, 26(7), 341-354. doi:10.5232/ricyde2011.02601

Martínez, J. R., Guillén, F., \& Feltz, D. L. (2011). Psychometric properties of the spanish version of the collective efficacy questionnaire for sports. Psicothema, 23(3), 503-509.

Martínez, R., \& Ciruelos, O. (2013). Collective efficacy, cohesion and performance in Spanish amateur female basketball. Revista de Psicología del Deporte, 22(1), 235-238.

Magyar, T. M., Feltz, D. L., \& Simpson, I. P. (2004). Individual and crew level determinants of collective efficacy in rowing. Journal of Sport and Exercise Psychology, 26, 136-153. doi.org/10.1123/jsep.26.1.136

Myers, N. D., Feltz, D. L., \& Short, S. E. (2004). Collective efficacy and team performance: A longitudinal study of collegiate football teams. Group Dynamics: Theory, Research, and Practice, 8, 126-138. doi:10.1037/1089-2699.8.2.126

Nunnally, J. C. (1978). Psychometric theory (2nd ed.). New York: McGraw-Hill.

Öncü, E., Feltz, D. L., Lirgg, C. D., \& Gürbüz, B. (2018). The examination of the psychometric properties of the Turkish Collective Efficacy Questionnaire for Sports. Acta Gymnica, 48(1), 27-35. doi:10.5507/ag.2018.005

Paes, M. J. (2014). Validação do collective efficacy questionnaire for sports (CEQS) para atletas brasileiros (Unpublished master thesis). Universidade Federal do Paraná, Curitiba.

Petitta, L., \& Jiang, L. (2015). The Differential mediating roles of task, relations, and emotions collective efficacy on the link between dominance and performance: A multilevel study in sport teams. Group Dynamics: Theory, Research, and Practice, 19(3), 181-199. doi.org/10.1037/gdn0000031 
Short, S. E., Sullivan, P., \& Feltz, D. L. (2005). Development and preliminary validation of the Collective Efficacy Questionnaire for Sports. Measurement in Physical Education and Exercise Science, 9(3), 181-202. doi.org/10.1207/s15327841mpee0903_3

Uchida, R., Machida, M., Tsuchiya, H., \& Kugihara, N. (2014). Revision and japanese translation of the collective efficacy questionnaire for sports: Test of its construct validity. Japan Journal of Physical Education, Health and Sport Sciences, 59(2), 841-854. doi.org/10.5432/jjpehss.13086 